\title{
Research and Application of Online Monitoring Fault Diagnosis for Infrared Image and Visible Image Registration
}

\author{
Jianqiang Huang ${ }^{+}$, Huan Wu, Xiaoying Wang and Zhengming Yi \\ State Key Laboratory of Plateau Ecology and Agriculture, Department of Computer Technology and \\ Application, Qinghai University, Xining 810016, Qinghai, China.
}

\begin{abstract}
To find the hidden trouble in substation accident early, it is necessary to build a infrared temperature monitoring and warning system, which can collect infrared image of substation electrical equipment automatically and circularly, through extracting the temperature information of the objective device to distinguish fault. In order to solve the positional deviation of the image, which caused by the mechanical transmission, leads to that it is difficult for systems to monitoring and positioning problem on interested equipment, so it needs to use corrected method of image registration, firstly segmenting object and defining configuration for image, and extracting the target which is detected, using suitable image registration method to register the deviation between a sequence of image. So that the system can get the temperature of the equipment parts in time and accurately, and ensure the reliable operation of the monitoring system. The infrared image and the visual image registration need to be registered and the fault region in the visible light image need to be marked for detecting the fault zone accurately. In the implementation, we use the matching method for the registration of infrared image and visible light image which base on the trunk line pair ofreference ${ }^{1}$.
\end{abstract}

Keywords: infrared temperature monitoring, positional deviation, image registration, trunk line pair.

\section{Image registration}

Image registration is a process that stack images which obtained by a different period, different viewpoints or different sensors in the same region or objects up, its main purpose is to eliminate or reduce the geometric deformation which caused by different imaging conditions between fiducial image and to be corrected image, so as to obtain two images of geometric consistency.1 The purpose of image registration is getting mapping that the image to be registered (in this paper, refers to the infrared image) relative to a fiducial image (in this paper, refers to the visible image) in space and intensity. Using $\mathrm{T}$ (x,y) and B (x, y) said the image to be registered and the fiducial image, then the mapping relationship can be expressed as: $\mathrm{B}$ $(\mathrm{x}, \mathrm{y})=\mathrm{g}(\mathrm{T}(\mathrm{f}(\mathrm{x}, \mathrm{y})) 2$, in which the $\mathrm{g}$ is the gray-scale transformation, the $\mathrm{f}$ is the coordinate transformation of the two-dimensional geometry space.

Since this paper is mainly concerned on location matching of the infrared image and visible light image in geometric space. Therefore, the mapping can reduced to $\mathrm{V}(\mathrm{x}, \mathrm{y})=\mathrm{I}(\mathrm{f}(\mathrm{x}, \mathrm{y}))$. Among them, $\mathrm{V}(\mathrm{x}, \mathrm{y})$ said visible light images, $\mathrm{I}(\mathrm{x}, \mathrm{y})$ said infrared image.

In order to obtain the mapping relationship, it is necessary for infrared image and visible image to feature extraction and matching, so as to obtain corresponding points to set of two images, and further calculating the specific mapping function through matching points to set. The image matching process usually includes pre-processing, feature extracting, feature matching, optimization and etc. (Fig.1).

\footnotetext{
+ Corresponding author. Tel.: +86 15509789239.

E-mail address: hjqxaly@163.com.
} 


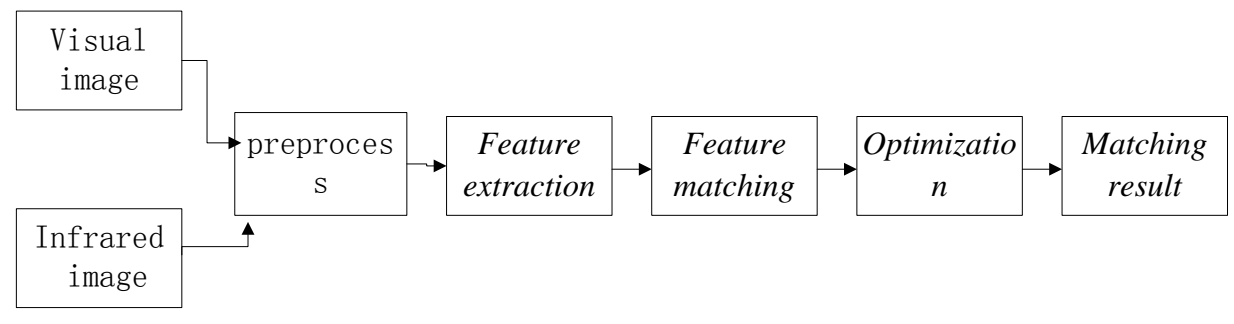

Fig. 1: Image matching process.

\section{Realization of Image Registration System}

\subsection{Image registration process}

Image registration process is usually divided into pre-processing, feature extraction, feature matching, optimization and other steps. Among them, the pre-treatment part will sharpen, fuzzy or deblurring on the image, so as to make the image features more obvious. Feature extraction can be required from the infrared image and visible image features, as the material required for image matching. Feature matching part is matching the extracted features, so as to obtain the basic feature matching. Optimal subset in the optimization of processing is selected the matching from the resulting feature matching results, and get the final matching result accordingly. Among them, the greatest impact on the matching results is the feature extraction and matching part.

\subsection{Pretreatment}

The pre-processing part usually has sharpening, fuzzy, deblurring, binary dealing and other operations for original image, so as to prepare for feature extraction.

In the realization of this paper, the pre-treatment part includes three parts: deblurring, sharpening or fuzzy processing, gray-scale.

Deblurring: Due to visible light image in the original image has more obvious motion blur. Therefore, it is necessary for visible light image to deal with deblurring firstly. The basic deblurring algorithm use the fast non consistency deblurring algorithm in references3,4to remove dynamic fuzzy. Taking into account the actual system, the resulting image does not appear obvious dynamic fuzzy, so does not integrated this part into image registration system, but use the processing program which is provided in reference5.Need to pay attention to that deblurring operation remove large-scale motion blur which is caused by camera movement, jitter and other operation, but the fuzzy effect of fuzzy processing is a small-scale fuzzy (the aim to make the image feature is more coherent), the two parts have obvious differences.

Sharpening / fuzzy processing: According to the preset fuzzy check original picture, there are corresponding sharpening / fuzzy processing, the purpose of sharpening is that has more obvious specific characteristic, the vague purpose lies in obtaining line, contour and other continuous feature. In the current implementation, due to the visible light image is more ambiguous, dose not carry out this part of the processing.

Gray level: Gray-scale operations are that convert color images to gray-scale images.

\subsection{Feature extraction}

Feature extraction and matching is a key step in image registration. The common image features includes gray value, contour, corner points, lines, etc.1,2,6,7,8,9,10 These characteristics have their own advantages and disadvantages, among them, the most commonly used is the gray features, gray features can calculate quickly, but also has a high matching precision, so it in the field of conventional image registration has been usedwidely. However, in view of infrared image and visible light image registration, because there is a big different gray value between the infrared image and visible light images, the gray value feature does not apply. In reference8, the test show that, based on the gray feature of the famous sift matching algorithm, it is a complete failure in the infrared image and visible light image matching. Therefore, the registration of infrared image and visible image can only use the shape feature of contour, line, etc. Considering the complicated pipeline system in the high voltage devices, the horizontal and vertical pipeline system constitute the natural characteristics of the relationship in the image. Therefore, we use the straight line as the 
matching feature in this paper. In order to improve the matching efficiency and accuracy, we use the trunk line of reference 1 which is more able to reflect the structural characteristics of the image, as the selected features.

As the name suggests, the trunk line pair is a pair of lines with special features in the image. Trunk line pair is the straight line pair1 which meets the requirements that the image of the length, angle, and the distance. Specifically, these requirements include:

(i) Linear length not less thanl $\mathrm{th}_{\text {th }}$;

(ii) Angle which between straight lines approximate right angle;

(iii) Distance which between trunk lines is less than $\mathrm{D}_{\mathrm{th}} \cdot 1$

What can be seen from the definition that trunk line can reflect the image of the skeleton feature in a large extent, especially in the pipeline system, crossed pipeline image constitute the natural trunk line pair. So this paper uses the feature matching method based on the trunk line pair. Specific flow chart 2.

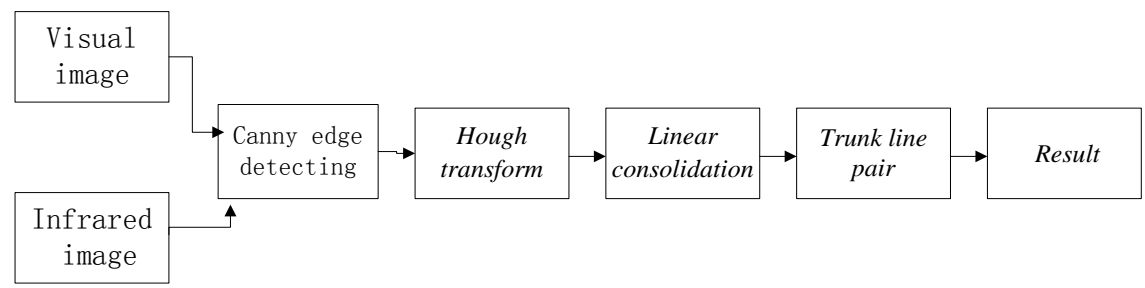

Fig. 2: Extraction process of trunk line.

Line detection: The prerequisite for obtaining the trunk line pair is to obtain the continuous line segment in the image. In this paper, the Hough transform, which is provided by the Open CV image processing library, extract the straight line in the image. Specific processes are as follows: Firstly extracting edge features of the image that after pre-processing through Canny operator. At the same time, the image is converted to a binary image, then extract continuous line from edge through Hough transformation, so as to obtain all line features in the image which meet the requirements.

Linear combination: After obtaining all straight lines which accord with the requirements, it is necessary for the lines which are parallel and close to merge and stitch, the line after stitching constitute linear feature set. Because that in infrared image or visible image which has motion blur, both sides of the cable are easily identified as two straight lines, not only influences the matching accuracy but also will increase the amount of computation. Therefore, it is necessary to stitch and merge. At the same time, the straight line is longer, easier to be recognized and more able to reflect the structure of the image after stitching.

Trunk line pair extraction: After the completion of the merger and the combination of straight line, it is necessary to extract the trunk line from the straight line. The specific extraction method of trunk line pair is shown in the 2.2 section of the reference1.The idea is: Traversing linear feature set, obtaining all straight lines which meet the length requirements, and the pairwise pairing, so that obtain preliminary trunk line pair on the set. Then we traverse trunk line pair on the set, calculating distance and angle between each pair of straight line, so as to get usable trunk line pair on the set.

\subsection{Feature extraction}

Aiming at the infrared image and visible image to extract trunk line pair respectively, can obtain the two corresponding sets of trunk line pair. The next critical step is to match the sets of trunk line pair in two images and use the vertex which match the trunk line pair to the corresponding(intersection of two lines in trunk line pair) as the basis of computing and transformation on the model $\mathrm{f}$.

The original matching algorithm in reference1, solely using the difference of included angles between two pairs of trunk line pair (defined as trunk the angle of two lines) as the based on feature matching. This advantage is that it can overcome the deviation which caused by the rotation transformation, the disadvantage is that, the matching that we get lack of location information and dose not accurate enough so as to further increase the partial optimal computation. 
Taking into account on the online detection system, there is no serious rotation and distortion between the infrared and visible light image, the two pieces of the image on the left and right and up and down range is consistent basically and the center points of the two images will not have serious deviation. Therefore, in the process of feature matching, using the position reference information as matching conditions further. Specific content as follows:

(i) The included angles between two pairs of matching trunk lines should be less than the specified value of $\theta_{1}$;

(ii) The coordinate system is constructed with the image center as the origin, and the vertex of two matching trunk lines should be located in the same quadrant;

(iii) In the coordinate system, the distance between the vertex of two matching trunk lines and point of the origin is proportional to the length of the picture.

The application of the matching rule can get the matching trunk line pair of the set between infrared image and visual image.

\subsection{Optimization}

Though adding information about the relative position, because the final transformation model were obtained before, unable to get accurate position, it is rough for relative position information of the threshold to set, before the calculation of the final transformation model, it is necessary for the set of matching which get already to optimize. In addition, the matching trunk line pair of the set contains all trunk line pair of the set which meet the conditions of threshold, will lead to the duplication and conflict inevitably, the matching of repetition and conflict needs to be resolved and correction.

Conflict and duplication is easy to solve, just in the process that select required trunk line pair from the matching trunk line pair of the set deleting repeat and the conflict. The focus of this part is still optimization, because the optimal results will affect the final result directly.

Using the optimal and objective function in the implementation, further adjustment and optimization are based on the optimal and objective function in reference 1.

As for the matching trunk line pairs (denoted as $\mathrm{ML}_{1}$ and $\mathrm{ML}_{2}$ ), using the matching degree of the functions to mark them as flow, $\mathrm{MD}\left(\mathrm{ML}_{1}, \mathrm{ML}_{2}\right)=\frac{1}{0.1+\left(\theta_{1}-\theta_{2}\right)}+\frac{1}{0.1+\left(\mathrm{l}_{1}-\mathrm{l}_{2}\right)}$. Among them, $\theta$ is the included angle between the trunk line pair and 1 is the sum of the lengths of the two straight lines in the trunk line pair.

Denoting that the matching value to be MD, and the matching degree of the matching trunk line pair of the set is defined as:matchValue $=\sum_{\mathrm{i}=0}^{\mathrm{M}} \mathrm{MD}_{\mathrm{i}}-\mathrm{ERR}$; Among them, $\mathrm{M}$ is the number of matching pairs in the set, ERR is defined as error, which setting all the matching trunk line pair of the vertices as parameters, then calculating error that the transformation model on the original input from least squares method.

After getting the matching degree of set of trunk line pair, just as the objective function, selecting the matching subset from the set of matching trunk line pair can let the value of objective function be maximums. Due to the objective function of ERR is determined after that the subset is determined. Therefore, the optimal problem is a NP problem which lack of exact solution of polynomial time.

In this implementation, testing the branch and bound method and genetic algorithm as the effect of optimal algorithm. And the genetic algorithm is adopted in the end.

Branch and bound method can obtain the exact solution, but due to the need for objective function to calculate accurately at the end of the branch, therefore it can not be effective pruning, the computation time is too large, therefore, in the actual implementation, selecting the set of initial solution of branch and bound from the MD value of the fifteen largest solution group. This can reduce the running time of the optimization part at a great extent, but relatively, the result is not accurate enough.

Considering the defects of the branch and bound method, further introducing genetic algorithm, the genetic algorithm is a heuristic algorithm, cannot obtain the exact solution, but there is great probability to obtain the feasible solution. Simply because the MD values will lead to that the matching information of position is missing, seriously influence the accuracy of result, so compared to the simplified branch and bound method, genetic algorithm is more efficient. 


\subsection{Transformation model}

Considering the characteristics of the online detection system, between the infrared and visible images, only have transformation that include simple translation, scaling, cropping, etc. It will not be complex distorted and the turnover. Therefore, the simple affine transformation is sufficient to describe the relationship between the two images accurately.

Therefore, between the infrared image and the visible image should meet the affine transformation model:

$$
\left\{\begin{array}{l}
\mathrm{x}_{1}=\mathrm{a}_{1} \mathrm{x}_{2}+\mathrm{b}_{1} \mathrm{y}_{2}+\mathrm{c}_{1} \\
\mathrm{y}_{1}=\mathrm{a}_{2} \mathrm{x}_{2}+\mathrm{b}_{2} \mathrm{y}_{2}+\mathrm{c}_{2}
\end{array}\right.
$$

Among them, $\left(\mathrm{x}_{1}, \mathrm{y}_{1}\right)$ is the coordinate of the visible light image, $\left(\mathrm{x}_{2}, \mathrm{y}_{2}\right)$ is the coordinate of the infrared image.

Only need to use the least squares method base on the set of optimized matching trunk line pair to obtain six parameters, such as $a_{1}, a_{2}, b_{1}, b_{2}, c_{1}, c_{2}$, so as to complete matching.

After getting the complete matching model, there is an infrared image to visible light image mapping at a point and a straight line of arbitrary and arbitrary shape through the matching model.

\subsection{Results further optimization}

The optimization problem corresponding to the registration problem is NP problem, the solution space is very large, it is very difficult to obtain the exact solution. The main reason for this problem is that feature extraction and matching process lack of accurate location information, which may lead to false matches between trunk line pair that be composed by similar or adjacent lines, further led to the solution of optimization problems space complex. Therefore, if you can provide more accurate location information in advance, will enable the matching results optimized greatly.

A simple and easy method of obtaining the accurate location information is that obtain transformation model parameters between infrared image and visible light image through marking many points artificially in advance. Then in the matching process, the distance between the vertices of matching trunk line pair the vertex that calculated by mapping function to, which is limited within a certain error range.

Due to the detection system, it can obtain many infrared images and visible images continuously. Consider to that there is similar transformation relation between these images, therefore, only need to mark on a pair of infrared image and visible light image, so as to enhance the registration accuracy greatly.

\section{Test Result}

The test procedure of sample based on 2.4.11 OpenCV, using $\mathrm{C}++$ language. The test environment is Mac OSX Yosemite 10.10.4, IntelCore i5@2.6GHz Mac, the compiler is Apple LLVM 6.1.0 (LLVM 3.6.0). The running environment of deblurring program is R2015a Matlab.

\subsection{Deblurring results}

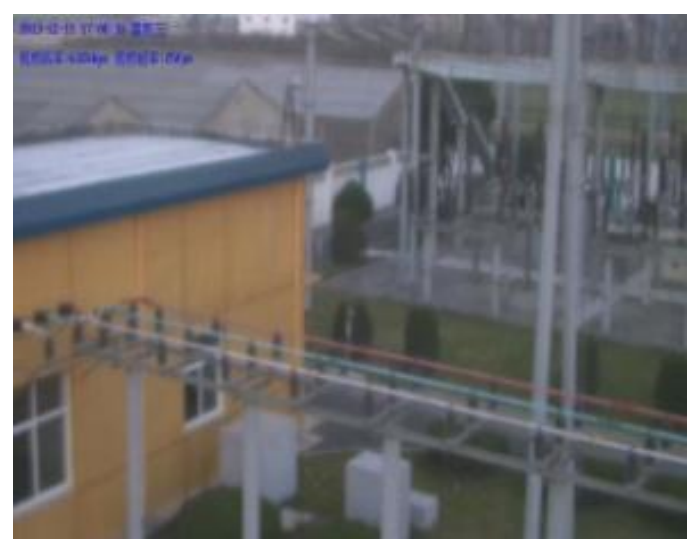

Fig. 3: Original image.

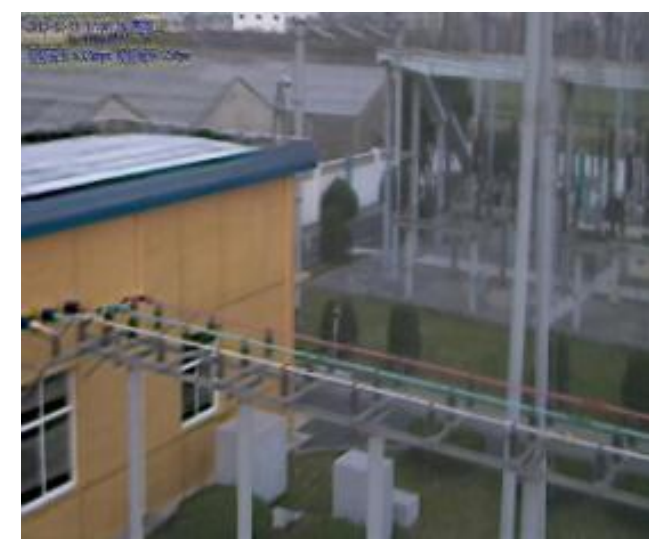

Fig. 4: Deblurring results.

\subsection{Match result}




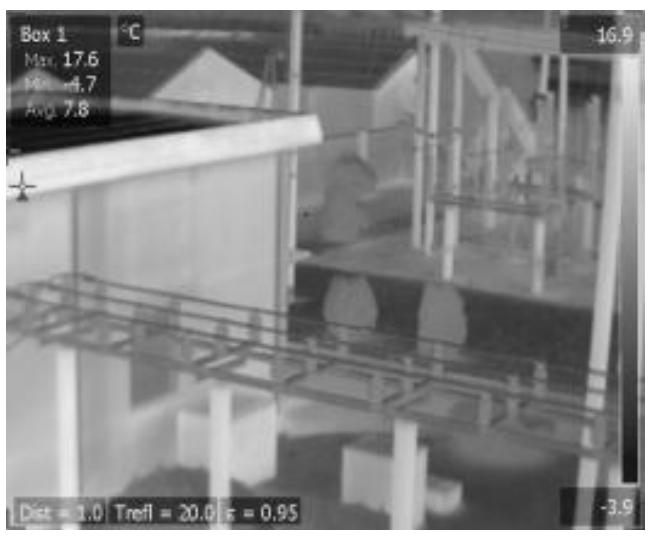

Fig. 5: Registering image 1.

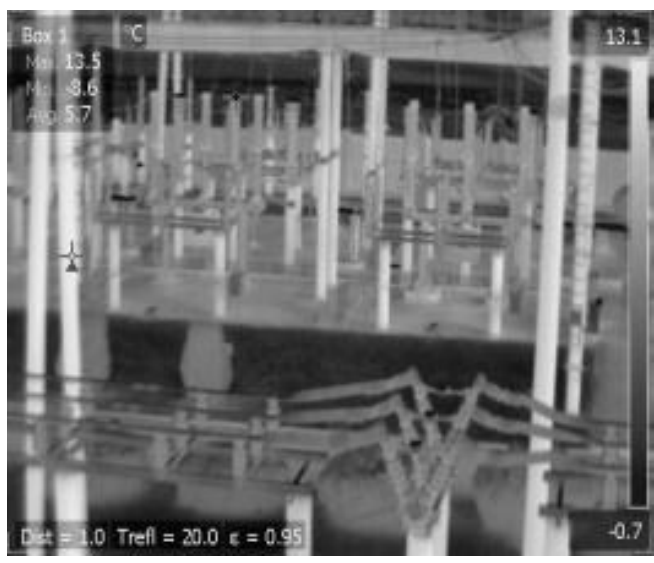

Fig. 7: Registering image 2.

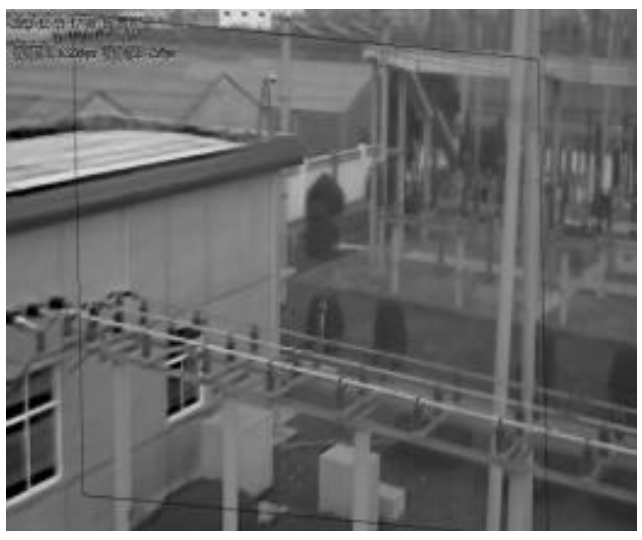

Fig. 6: Registration results 1.

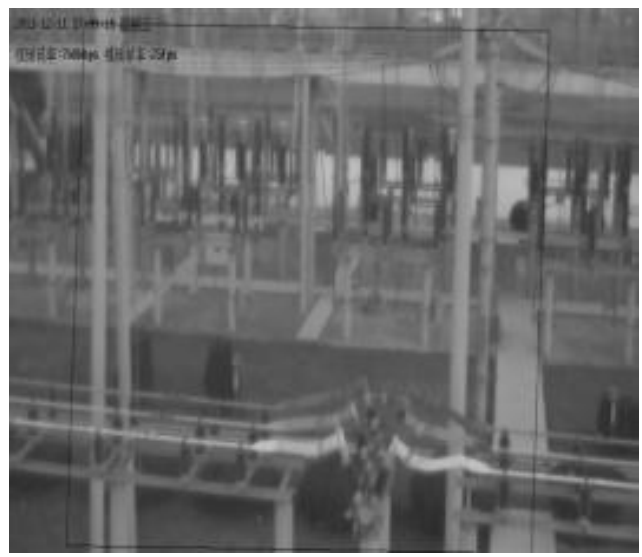

Fig. 8: Registration results 2.

In Fig. 6 and Fig. 8, there are black quadrilateral shapes, which are matching results of the whole no registration image (IR images) in the benchmark image (visible light image). It can be seen from the matching results, although there is a slight bias in matching results, marking note basically and accurately except the region corresponding to matching image. The main problem is that the rectangular area is matched to the parallel quadrilateral region. The main reason is that, due to the lack of accurate location information, getting feasible solution only through genetic algorithm, and in the feasible solution, often confusing similar straight line, which leads to that rectangular region is matched for parallelogram region.

It can also be found that, as for the matching of two different image pair, there are acceptable results. The examples in this paper are independent from the common registration image method.

\subsection{Labeled matching results}
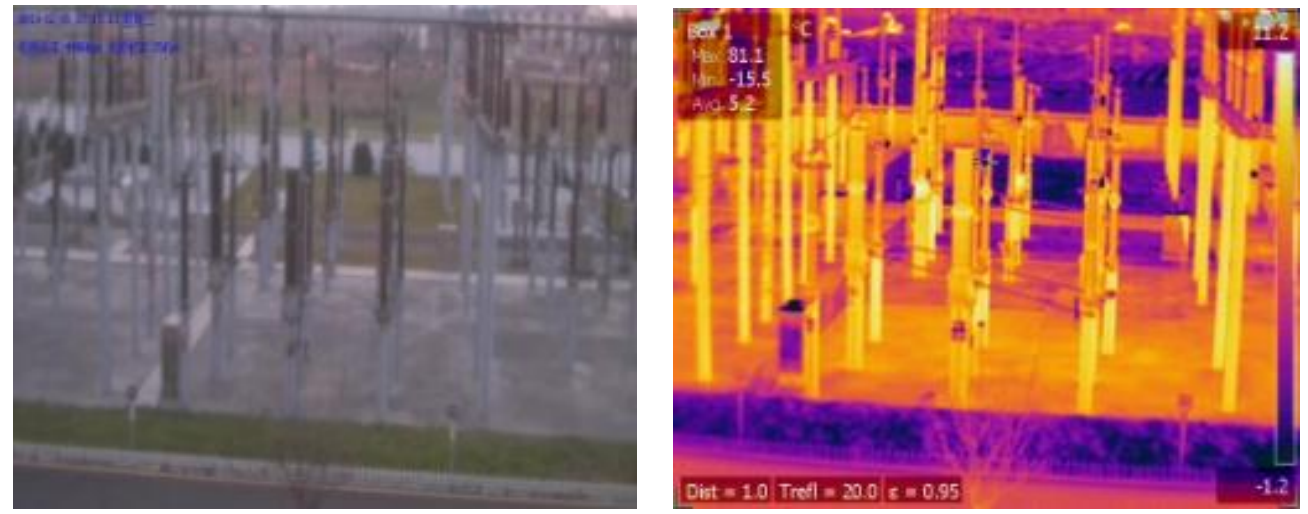

Fig. 10: Tagged images manually.

Compared to the previous no registration image, it can be found that, there are very accurate matching results through marking completely different but acquired by the same camera images. 


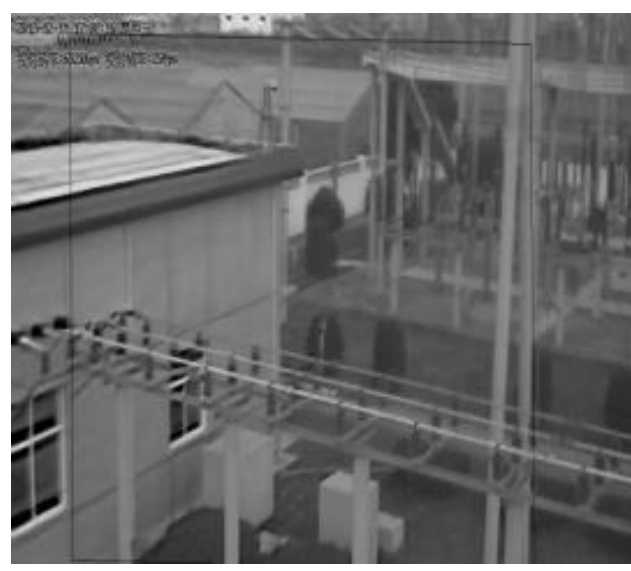

Fig. 11: Registration results 1 .

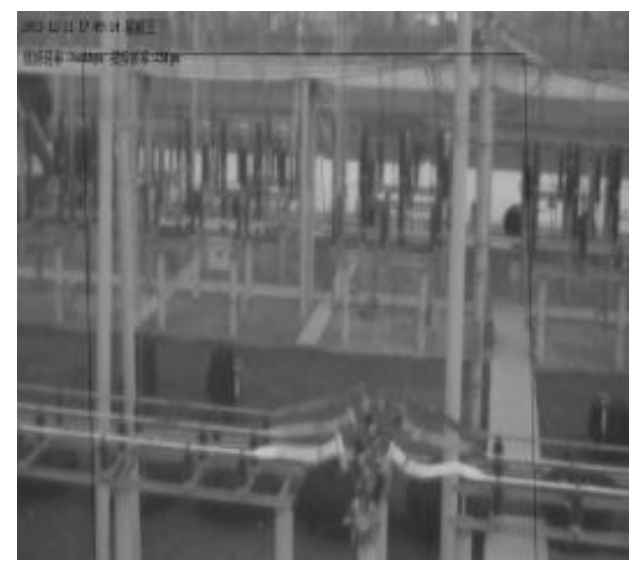

Fig. 12: Registration results 2 .

\section{Work to Be Carried out in the Future}

This paper realizes the registration algorithm of infrared image and visible image base on trunk line pair, and achieved good results. However, there are still some problems in the realization of this paper, which needs further work. In this paper, the problems are mainly concentrated in two aspects:

(i) Registration results are not accurate enough. The line matching is easy to cause the lack of locational information, the contour matching can make up for this problem in a certain extent. But at the beginning of the test, due to the visible light image is fuzzy, it is difficult to extract clear outline, so had to give up the assumption that use of contour matching cooperate with the linear matching. If may be available in the future to get more clear and original image or find a better deblurring algorithm, using contour matching to assist line matching, will get better results.

(ii) The efficiency of the optimization algorithm is low. In the genetic algorithm, the difference of matching value between the different results is too large, have to use the larger population. It leads to take a long period of time to run the algorithm requires and can consider to improve the efficiency of the implementation of the program by improving algorithm or parallel in the future.

\section{Summary}

This paper realizes the registration algorithm of infrared image and visible image base on the trunk line pair, and achieves good results. This paper gives the analysis of the concrete implementation process and the advantages and disadvantages of it, and put forward the direction and methods of further optimization.

\section{Acknowledgment}

The authors would like to thank the anonymous reviewers for their helpful advices to improve this paper. This work was supported in part by a grant from the Applied basic research in Qinghai Province of China(2014-ZJ-718) and Natural Science Foundation of China((61363019),(61440021)) and the Young and middle-aged Foundation Program of Qinghai University(2013-QGY-10),(2014-QGY-23).

\section{References}

[1] GaoFeng, Wen Gongxian,LvJinjian. Based on the trunk of the infrared and visible light optimal image registration algorithm [J]. Computer Journal of, 2007, 30 (6)1014-1021.

[2] Wang Kunpeng,XuYidan, Yu Qifeng. Infrared and visible light image registration method classification and the status quo [J]. Infrared technology, 2009 (5): 270-274.

[3] Whyte O, Sivic J, Zisserman A. Deblurring shaken and partially saturated images[J]. International Journal of Computer Vision, 2014, 110(2): 185-201.

[4] O. Whyte, J. Sivic, A. Zisserman, and J. Ponce. Non-uniform Deblurring for Shaken Images. IJCV, 2011

[5] http://www.di.ens.fr/willow/research/saturation

[6] Gao Jing, Sun Jiyin, Liu Jing. Based on gray level in the neighborhood information of Hausdorff distance image 
matching method [J]. Computer application, 2011, 31 (03): 741-744.

[7] Wang Ani, Ma Caiwen, Liu Shuang, etc. Based on the corner of infrared and visible light image automatic registration method. Acta PHOTONICA Sinica, 2009, 38 (12): 3328-3332.

[8] Cao Zhiguo, Yan Ruicheng, Song Zhe. Using fuzzy shape context of the infrared and visible light image matching method [J]. Infrared and laser engineering, 2008, 37 (6): 1095-1100.

[9] Wang Jialin, Yang Junjin. Study on fast matching algorithm [J]. automation technology and application of infrared image, 2009 (9): 76-78.

[10] Brown L G. A survey of image registration techniques[J]. ACM computing surveys (CSUR), 1992, 24(4): 325-376. 